\title{
Incremental Service Composition Based on Partial Matching of Visual Contracts
}

\author{
Muhammad Naeem ${ }^{1}$, Reiko Heckel ${ }^{1, \star}$, Fernando Orejas ${ }^{2, \star \star}$, \\ and Frank Hermann ${ }^{3}$ \\ 1 Department of Computer Science, University of Leicester, United Kingdom \\ $\{$ mn105, reiko\}@mcs.le.ac.uk \\ 2 Departament de L.S.I., Universitat Politècnica de Catalunya, Barcelona, Spain \\ orejas@lsi.upc.edu \\ 3 Fakultät IV, Technische Universität Berlin, Berlin, Germany \\ frank.hermann@tu-berlin.de
}

\begin{abstract}
Services provide access to software components that can be discovered dynamically via the Internet. The increasing number of services a requester may be able to use demand support for finding and selecting services. In particular, it is unrealistic to expect that a single service will satisfy complex requirements, so services will have to be combined to match clients' requests.

In this paper, we propose a visual, incremental approach for the composition of services, in which we describe the requirements of a requester as a goal which is matched against multiple provider offers. After every match with an offer we decompose the goal into satisfied and remainder parts. We iterate the decomposition until the goal is satisfied or we run out of offers, leading to a resolution-like matching strategy. Finally, the individual offers can be composed into a single combined offer and shown to the requester for feedback.

Our approach is based on visual specifications of pre- and postconditions by graph transformation systems with loose semantics, where a symbolic approach based on constraints is used to represent attributes and their computation in graphs.
\end{abstract}

\section{Introduction}

Service-oriented Architecture (SOA) 1] supports dynamic discovery and binding based on matching requesters' requirements with providers' offers. Both requirements and offers can be expressed as specifications of the (expected or given) semantics of a service's operations in terms of their pre- and postconditions. At a technical level this is supported by semantic web technologies (e.g. OWL-S [2], WSML [3]), at modeling level visual contracts have been suggested to describe service semantics [4.

\footnotetext{
* Partially supported by the project Sensoria IST-2005-016004.

** Partially supported by the MEC project (ref. TIN2007-66523), the MEC grant PR2008-0185, the EPSRC grant EP/H001417/1 and a Royal Society travel grant.
} 
However, expecting to find a single service for each requirement is unrealistic. Often services need to be combined to satisfy the demands of clients. For example, let us consider a scenario, where a requester is looking to book a trip for attending a conference. The requester may be interested in flight and hotel reservation. Rather than using a single service, the requester may have to use two separate service providers.

In this paper we propose an incremental approach for service composition, where we assume that the requirements are expressed by a single goal stating pre- and postconditions. A variety of offers could contribute to the goal, each described by pre- and postconditions as well. We propose a notion of partial matching of offers with goal. After every partial match we compute the remaining requirements by decomposition of the original goal into the satisfied subgoal and its remainder. We iterate this process until the goal is achieved or we do not have any more offers.

As a result of this procedure we produce a combined offer which can be visualized and reviewed by the client. Our approach thus supports semi-automatic service composition.

\section{Related Work}

As motivated above, we work on the assumption that it is unrealistic to expect a single offer to be sufficient to satisfy a goal, i.e., several offers will have to be combined. This raises the first three of the following questions to serve as criteria for approaches to dynamic service composition. The fourth question derives from the desired integration into mainstream modelling techniques such as the UML, which use diagrammatic languages to specify software. For service specification to be integrated into standard software engineering processes, they have to use compatible visual notations.

- Partial Match: Does the approach support partial matching of an offer with a goal, or is full satisfaction of all requirements necessary for each match?

- Flexibility: Does the approach allow to match offers in flexible order or does it follow a given control flow?

- Completeness: Is the approach decidable, i.e., does it provide a complete and terminating procedure to find out if there are combinations of offers satisfying a goal?

- Visualisation: Does the approach provide a visual language for service specification and feedback on the result of the matching?

While there are many approaches to composition of services, we limit our discussion to semantics-based approaches using pre- and postconditions, disregarding process or workflow-based orchestration, see [5]6] for a more complete picture. We summarise the results of our analysis in Table 1.

In [7] the authors propose a semi-automatic approach for filtering and composition of services using OWL and DAML-S. An inference engine performs an exact match with available services and shows the resulting list to the user who 
Table 1. How existing approaches realize the proposed requirements

\begin{tabular}{|c|c|c|c|c|c|}
\hline Approach of: & Language & Partial Match & Flexibility & Completeness & Feedback \\
\hline 7 & OWL, DAML-S & $\checkmark$ & $\bar{x}$ & $\bar{x}$ & $\sqrt{ }$ \\
\hline 8 & DAML-S, SHOP-2 & $\checkmark$ & $\checkmark$ & $\checkmark$ & $x$ \\
\hline 9 & FOL & $\checkmark$ & $\checkmark$ & $x$ & $x$ \\
\hline 10 & WSML & $\checkmark$ & $\checkmark$ & $\checkmark$ & $\times$ \\
\hline Our Approach & Graph Theory, GTS & $\checkmark$ & $\checkmark$ & $\checkmark$ & $\checkmark$ \\
\hline
\end{tabular}

selects the ones to be composed. The approach is highly dependent on user input and so avoids the need for a decidable composition procedure required for automation. In contrast, we would require feedback on the end result of the automated composition only.

In a number of works, AI planning models are used to construct process models from goals and operations described by pre- and postconditions. For example, 8] is based on DAML-S. Their approach is decidable for a finite number of services / operations. Partial matching is possible based on the semantic description. The main difference with our approach is the use of logic-based (rather than visual) descriptions, which makes it difficult to provide feedback on the result of the composition to domain and business experts.

The work in 9] is representative of approaches based on first-order specification of goals and services. It allows partial matching and flexibility in ordering in addition to goal templates which abstract from the actual input parameters for invoking services and can thus be matched at design time. Our goals are at the level of goal templates in [9] in that they are generic with regard to the actual parameters.

Approaches such as 10 use semantic service web markup languages such as WSML-MX that are both specialised for the task of service description and matching and limited in expressiveness to guarantee computability.

\section{Graphical Service Specification and Matching}

Following 411, in this section we review the basic notions of service specification and matching used in the rest of the paper.

Visual Service Specification. According to [12, a web service describes a collection of operations that are network accessible, each specified by a pre- and a postcondition. As usual, a precondition denotes the set of states where that operation is applicable and the postcondition describes how any state satisfying the given precondition is changed by the operation. In our case the states can be seen as typed attributed graphs. This means graphs that may include values (the attributes) in their nodes or edges, all typed by a fixed type graph. For instance, Fig. 1 describes the type graph of the running example of a travel agency that we will use along the paper and Fig. 3 is an example of an attributed (instance) graph typed over that type graph after booking a flight. 


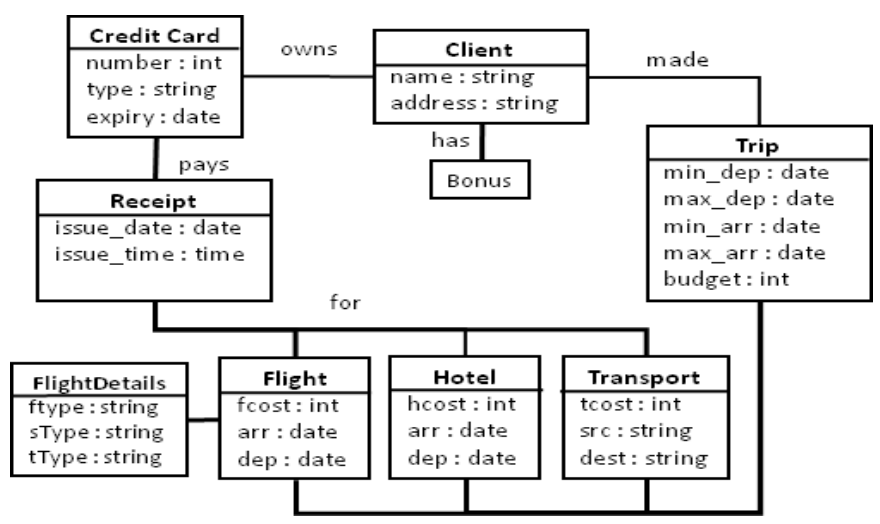

Fig. 1. Type Graph
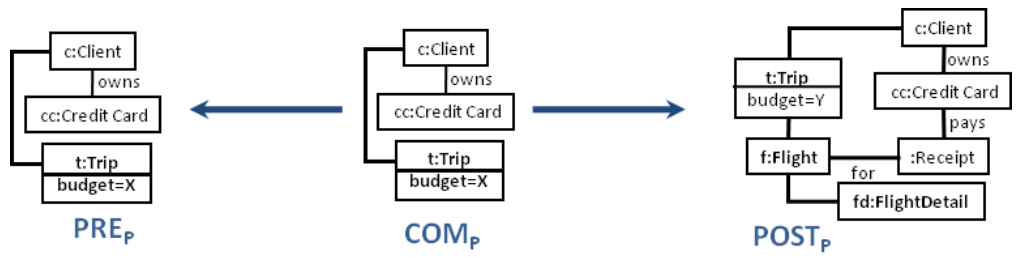

Conditions $(\alpha)$ :

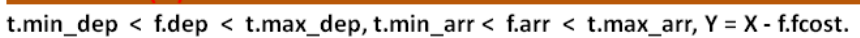

Fig. 2. BookFlight Operation for Travel Agency (Provider)

In 411] the specification of an operation $O p$, denoted $O p: P R E \Rightarrow P O S T$ is given by typed attributed graphs, $P R E$ and $P O S T$ related by an injective partial graph morphism represented as a pair of injective morphisms, pre :COM $\rightarrow$ $P R E$ and post : $C O M \rightarrow P O S T$. Here COM provides the intersection of $P R E$ and POST, and pre, post are the corresponding injections into PRE and POST. Usually, attributed graphs $P R E, P O S T$ and $C O M$ include variables, values or complex expressions as attributes. However, in this paper attributes will be restricted to variables and basic values only, ruling out complex expressions. These will be captured, together with other constraints, by a formula $\alpha$ which constrains the possible values of the variables occurring as attributes in a graph $G$ and we call $(G, \alpha)$ a constrained graph. In our case the graphs of an operation have a common condition $\alpha$ relating the variables occurring in PRE and POST. Hence, an operation specification is denoted by a pair $\langle P R E \Rightarrow P O S T, \alpha\rangle$ (or $\langle P R E \stackrel{\text { pre }}{\leftarrow} C O M \stackrel{\text { post }}{\rightarrow} P O S T, \alpha\rangle$ if we are interested in the intersection $C O M)$. For instance, Fig. 2 describes an operation BookFlight for booking a flight with a travel agency service.

In the example, graphs $P R E_{P}$ and $P O S T_{P}$ are the pre- and postconditions for operation BookFlight whereas graph $C O M_{P}$ represents the intersection of $P R E_{P}$ and $P O S T_{P}$. Condition $\alpha$ constrains the operation to be flexible enough 
to choose the departure date $f$.dep within the range of t.dep_min and t.dep_max as well as the arrival date f.arr within t.arr_min and t.arr_max. This way of dealing with attributed graphs, introduced in [13, has proved essential in order to support the declarative (as opposed to computational) description of attribute operations appropriate to the use of graphs as pre- and postconditions (rather than rewrite rules only). In particular, we allow attributed graphs representing states to include variables and conditions on attributes, rather than just values, thus providing a symbolic representation where not all the attributes are fully evaluated. We refrain from the use of application conditions 14 in the specification of operations to keep the presentation simple, but believe that they would not add an essential difficulty.

The semantics of the operation $\langle P R E \stackrel{\text { pre }}{\leftarrow} C O M \stackrel{\text { post }}{\longrightarrow} P O S T, \alpha\rangle$ is described using graph transformation. More precisely, given an attributed graph $\left\langle G, \alpha^{\prime}\right\rangle$ representing the current state and given a matching morphism $m: P R E \rightarrow G$ such that $\alpha^{\prime}$ implies $m(\alpha)$, with $m(\alpha)$ the formula obtained by replacing each variable $X$ in $\alpha$ by its image $m(X)$, the result will be the attributed graph $\left\langle H, \alpha^{\prime}\right\rangle$ with $H$ defined by the following double pushout:

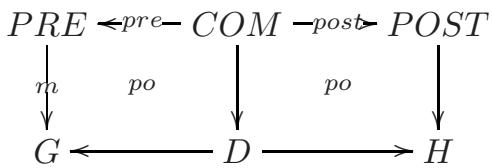

Intuitively, graph $D$ is obtained by deleting from $G$ all the elements (nodes, edges, or attributes) which are matched by an element that is present in PRE but not in $C O M$. Then, the graph $H$ is obtained adding to $D$ all the elements which are present in POST, but not in COM. For instance, if we apply the operation BookFlight to graph $G$ in the left of Fig. 3 , the result will be the graph $H$ in the right of Fig. 3. Notice that, for readability, these graphs include some values, such as 200, instead of a variable $X$ and the equality $X=200$ included in the associated formula.

A requester looking for a service must specify the operations they want to use. Requester specifications have the same form as provider specifications. They are seen as inquiries that the requester is making, with the aim of entering into a contract with the provider. In particular, $P R E$ would denote the data and resources that the requester would accept to provide and POST would describe the expected result of the operation. In this use case, the semantics of these

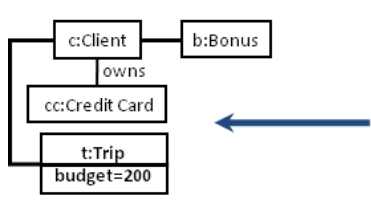

G

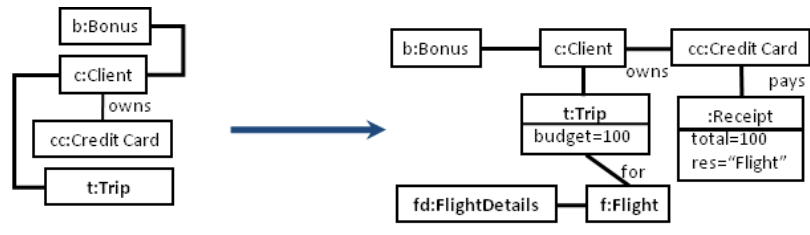

$\mathrm{H}$

Fig. 3. Transformation due to BookFlight of Travel Agency 
specifications is a different one because the requester may not (need to) know all the details of the provider state and cannot thus describe completely all the changes caused by the operation. Such a semantics has been studied for graph transformations in terms of double pullbacks [15]. More precisely, given an attributed graph $\left\langle G, \alpha^{\prime}\right\rangle$ and a matching morphisms $m: P R E \rightarrow G$ such that $\alpha^{\prime}$ implies $m(\alpha)$, a graph $H$ is the result of a double pullback transition if we can build a double pullback diagram of the same shape like the double pushout above, but replacing the po by $p b$ squares.

Intuitively, in a double pullback transition, the rule $P R E \Rightarrow P O S T$ describes a lower bound to the effects the operation should cause when applied to $G$. This means, if an element $a$ is present in PRE but not in $C O M$, then $m(a)$ must be deleted from $G$, and if $a$ is present in POST, but not in $C O M$, then it must be added to $G$. And if $a$ is present in $C O M$, then it must remain unchanged. However, $G$ may suffer other changes not specified by the operation. For instance, if the specification of the operation BookFlight in Fig. 2 would be part of a requester specification, then applying that operation to the graph $G$ in Fig. 3 could yield the graph $H$ in Fig. 3 as a result of a double pullback transition.

Matching Visual Contracts. In order to match the requirements for an operation $O p_{R}=\left\langle P R E_{R} \Rightarrow P O S T_{R}, \alpha_{R}\right\rangle$ of a requestor against a description $O p_{P}=$ $\left\langle P R E_{P} \Rightarrow P O S T_{P}, \alpha_{P}\right\rangle$ supplied by a provider, we have to guarantee that all effects required by $O p_{R}$ are implemented by $O p_{P}$. More precisely, assuming that $\langle G, \alpha\rangle$ represents the given state, the following conditions must be satisfied:

a) Whenever a transition for $O p_{R}$ can take place, a corresponding transformation associated to $O p_{P}$ must be possible.

b) If $O p_{R}$ prescribes that some element must be deleted from the current state, that element must also be deleted by $O p_{P}$.

c) If $O p_{R}$ prescribes that some element must be added to the current state, that element must also be added by $O p_{P}$.

d) If $O p_{R}$ prescribes that some element remain in the current state, that element should be part of $C O M_{P}$.

Technically, this means to ask for the existence of three injective morphisms $h_{P R E}: P R E_{P} \rightarrow P R E_{R}, h_{C O M}: C O M_{R} \rightarrow C O M_{P}$, and $h_{P O S T}: P O S T_{R} \rightarrow$ $P O S T_{P}$, such that $\alpha_{R}$ implies $h_{P R E}\left(\alpha_{P}\right), h_{P R E}$ and pre $_{R}$ are jointly surjective 1 , diagram (1) commutes, and diagram (2) is a pullback.

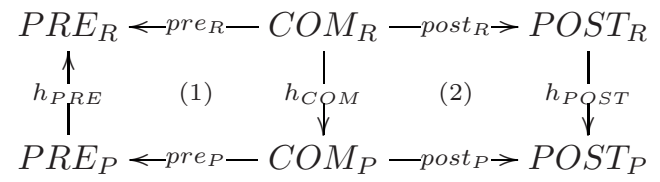

In particular, given $\langle G, \alpha\rangle$, the existence of $h_{P R E}: P R E_{P} \rightarrow P R E_{R}$ such that $\alpha_{R}$ implies $h_{P R E}\left(\alpha_{P}\right)$ ensures that if there is a match $m: P R E_{R} \rightarrow G$

${ }^{1}$ This means that every element in $P R E_{R}$ is the image of an element in $C O M_{R}$ or of an element in $P R E_{P}$. 


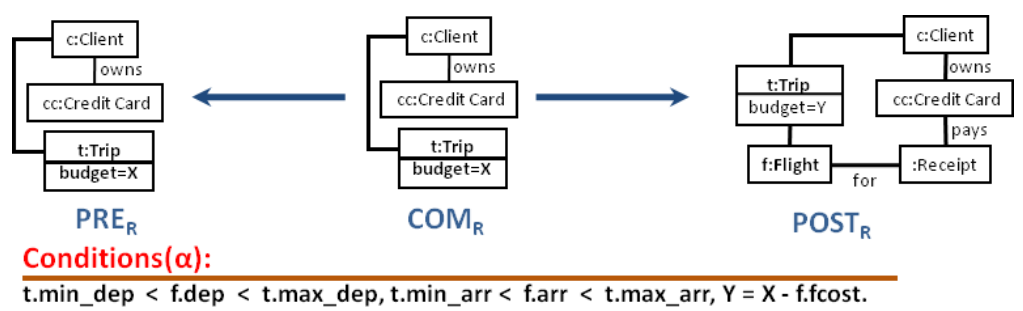

Fig. 4. BookFlight Operation of Requester

such that $\alpha$ implies $m\left(\alpha_{R}\right)$ then we also have a corresponding match $h_{P R E} \circ m$ : $P R E_{P} \rightarrow G$ such that $\alpha$ implies $h_{P R E}\left(m\left(\alpha_{P}\right)\right)$. In addition if an element is in $P R E_{R}$ but not in $C O M_{R}$, that element should also be in $P R E_{P}$, because $h_{P R E}$ and pre $_{R}$ are jointly surjective, but not in $C O M_{P}$ since diagram (1) commutes. Then, according to both rules, $O p_{R}$ and $O p_{P}$, that element must also be deleted. If an element is in $P O S T_{R}$ but not in $C O M_{R}$ then that element should also be in $P_{P S T_{P}}$ but not in $C O M_{P}$ because diagram (2) is a pullback. Finally, if an element is in $C O M_{R}$ its image through $h_{C O M}$ would also be in $C O M_{P}$.

In our example the specification in Fig.2 matches the specification in Fig. 4. All the elements in $P R E_{P}, C O M_{R}$, and $P O S T_{R}$ have an image in $P R E_{R}, C O M_{P}$, and $P O S T_{P}$ respectively. So there exist three injective morphisms 1614 between $P R E_{P}$ and $P R E_{R}, C O M_{P}$ and $C O M_{R}$, and $P O S T_{R}$ and $P O S T_{P}$.

We have discussed how to match a request with a service offered by a provider in an ideal situation, where the granularity of requirements and offered services coincide and the matching is complete. However, such a lucky outcome is unlikely in practice.

On one hand, as seen above, a service precondition must describe the data and resources that may be needed to run that service (and, in addition, through the associated condition $\alpha_{R}$, it may also describe the conditions under which a given service is considered to be acceptable, e.g. its cost). This means to assume that the requester knows, a priori, all the data and resources that may be required to satisfy his needs. This may be unrealistic in many cases. For instance, when booking a trip, the requester may describe in its precondition some basic data, like their name, date and destination of the travel, credit card or bank account number, etc. In addition, the requester may specify an overall budget for the travel. However the provider may also need to know the age of the traveller, to see if some discount applies, or whether the requester has a discount bonus that would be consumed when using the service.

On the other hand, the postcondition describes the effect of using a given service. In this sense, the requester will describe everything they expect to get when binding to a certain service. However, there may be two problems here. First, there may not be a single provider that offers a service covering all the requester needs. For instance, the requester for a travel may want, not only to book a flight and a hotel, but also to get tickets for a play and to have a dinner in a well-known restaurant. Then, there may be no travel agency that can take 
care of all these activities. And, second, the specification level of the requester and the provider may have different granularity. In particular, a requester may describe as a single operation booking a flight and a hotel room, while a given provider, in his specifications, may consider these two bookings as independent operations. Then, matching this request would mean for that provider finding an appropriate combination of the two operations that satisfies the customer needs.

So, we believe there is a need for matching a request with multiple offers. In the next section we discuss such an incremental procedure for the composition of services, where we will discuss the partial match of single requester operation with multiple provider offers.

\section{Incremental Service Composition}

Given a goal of a requester as well as a set of provider offers, both expressed by pre- and postconditions, first we select an offer providing a partial match of the goal. Then, we compute the remainder of the goal with respect to this offer, containing all the requirements not yet satisfied, and post the result as a new goal. We iterate these steps until all requirements are satisfied or we run out of offers to match. Finally, we compose all offers used into one global offer summarising the overall effect of the combined services. Next we describe this approach in detail.

Partial Matching. Given a request $\left\langle P R E_{R} \Rightarrow P O S T_{R}, \alpha_{R}\right\rangle$ a partial match with a provided description $\left\langle P R E_{P} \Rightarrow P O S T_{P}, \alpha_{P}\right\rangle$ is given by a partial embedding of $P R E_{P}$ into $P R E_{R}$ and of a partial embedding of $P O S T_{R}$ into $P O S T_{P}$. Following the previous discussion, the idea is that, on the one hand, not everything included in the provider's precondition needs to be present in the requester's precondition, since the latter may have to be completed later. On the other hand, not everything in the requester's postcondition needs to be present in the provider's postcondition, since not every effect demanded by the requester may be covered by a single provided operation.

Definition 1. (partial match, common suboperation) Given requester and provider operations $O p_{R}=\left\langle P R E_{R} \stackrel{\text { pre }_{R}}{\longleftarrow} C O M_{R} \stackrel{\text { post }_{R}}{\longrightarrow} P O S T_{R}, \alpha_{R}\right\rangle$ and $O p_{P}=$ $\left\langle P R E_{P} \stackrel{\text { pre }_{P}}{\longleftarrow} C_{P} \stackrel{\text { post }_{P}}{\longrightarrow} P O S T_{P}, \alpha_{P}\right\rangle$ a partial match $m$ consists of embeddings:

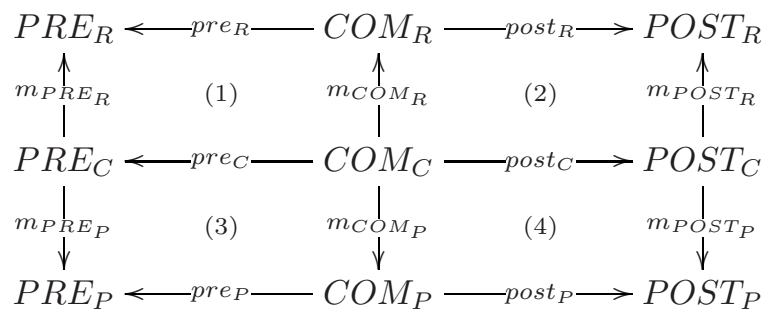

such that diagram (3) commutes, and diagrams (1), (2), and (4) are pullbacks. 


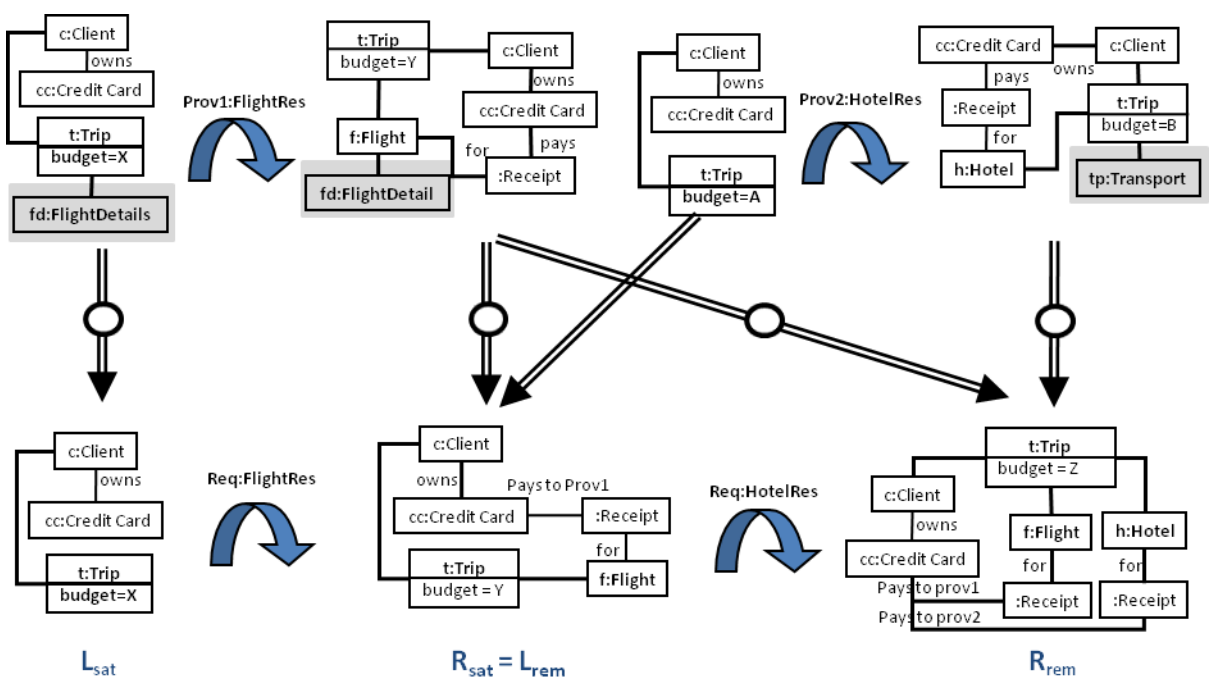

Constraints:

h.dep = f.dep,t.min_dep $\leq$ f.dep $\leq$ t.max_dep,t.min_dep $\leq$ h.dep $\leq$ t.max_dep, h.arr $=$ f.arr, t.min_arr $\leq$

f.arr $\leq$ t.max_arr, t.min_arr $\leq$ h.arr $\leq$ t.max_arr, $Y=X$ - f.fcost, $Z=Y-h . h \operatorname{cost}, B=A$ - h.hcost - tp.tcost.

Fig. 5. Requester goal jointly matched by two consecutive offers

The operation $O p_{C}=\left\langle P R E_{C} \stackrel{\text { pre }_{C}}{\longleftarrow} C O M_{C} \stackrel{\text { post }_{C}}{\longrightarrow} P O S T_{C}, \alpha_{C}\right\rangle$, where $\alpha_{C}$ is a condition such that $\alpha_{R}$ and $\alpha_{P}$ imply $m_{P R E_{R}}\left(\alpha_{C}\right)$ and $m_{P R E_{P}}\left(\alpha_{C}\right)$, respectively, is called a common suboperation of the provider and the requester operations, since it can be considered to be embedded in both operations. For example, the common suboperation is shown by unshaded background in Fig. 5 in both embeddings, where the circled arrows denote the partial embeddings between the providers and requesters pre and postconditions.

The condition $\alpha_{C}$ is obtained from $\alpha_{P}$ when some of its free variables are not present. In particular, $\alpha_{C}$ may be $\exists X \alpha_{P}$ or some stronger condition, where $X$ is the set of variables included in the provided operation which are not present in the common suboperation. The fact that we do not ask diagram (3) to be a pullback, while we ask diagrams (1), (2), and (4) to be so, is a consequence of the fact that we want to express the condition that $O p_{P}$ implements partially the effects of $O p_{R}$ on their common elements. This means, on one hand, that if a common element is deleted by $O p_{P}$ then that element must also be deleted by $O p_{R}$, but not necessarily the other way round. Conversely, this means that every common element preserved by $O p_{R}$ must also be preserved by $O p_{C}$ (and hence by $O p_{P}$ ), which means that $(1)$ is a pullback. However, the fact that not every common element preserved by $O p_{P}$ must also be preserved by $O p_{R}$ means that (3) is not necessarily a pullback. On the other hand, (2) and (4) are pullbacks, because we consider that if a common element in $P O S T_{C}$ is produced by $O p_{P}$, then it should also be produced by $O p_{R}$, and vice versa, That is, it makes no 
sense to think that an element that is considered to be information used by the requester's rule is produced by the provider's rule, or the other way round.

The common suboperation of $O p_{P}$ and $O p_{R}$, while being embedded into both operations, does not implement their common behaviour. When $O p_{C}$ is applied to a given state $\langle G, \alpha\rangle$, it adds all common elements added by both $O p_{P}$ and $O p_{R}$, but it only deletes common elements that are deleted by $O p_{R}$, but not necessarily by $O p_{P}$. For instance in Figure 5 the requestor goal requires the deletion of $b$ : Bonus but Prov1 :: FlightRes does not. The common suboperation of Prov1 :: FlightRes and Req :: FlightRes is constituted by all elements of Prov1 :: FlightRes not shaded in grey. Hence, $b:$ Bonus is in the precondition of the common common suboperation but not in the postcondition, i.e., it is deleted. However, we are interested in a common operation that describes the shared effects of $O p_{P}$ and $O p_{R}$, i.e., that deletes all elements deleted by both $O p_{P}$ and $O p_{R}$ and adds all elements added by both operations.

Definition 2. (shared behaviour suboperation) Given requester and provider operations $O p_{R}$ and $O p_{P}$, and given their common suboperation $O p_{C}$ with respect to a partial match $m$, we define the shared behaviour operation of $O p_{R}$ and $O p_{P}$ with respect to $m$ as $O p_{S B}=\left\langle P R E_{C} \stackrel{\text { pre }_{S B}}{\longleftarrow} C O M_{S B} \stackrel{\text { post }_{S B}}{\longrightarrow} P O S T_{S B}, \alpha_{C}\right\rangle$, where $C O M_{S B}$ and $P O S T_{S B}$ are given by the following pullback and pushout diagrams:
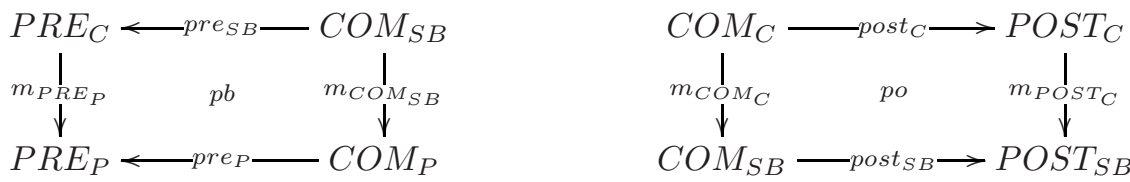

with $m_{C O M_{C}}$ defined by the universal property of the pullback defining $C O M_{S B}$.

Intuitively, $C O M_{S B}$ includes the elements which are shared by $P R E_{P}$ and $P R E_{R}$ and are not deleted by $O p_{P}$, and $P O S T_{S B}$ includes all the elements of $P O S T_{C}$ plus the elements that are not deleted by $O p_{S B}$. Fig. 6] depicts the shared behaviour suboperation based on the requestor goal and Prov1 :: FlightRes and their common sub operation (shown in Fig. 5 ).

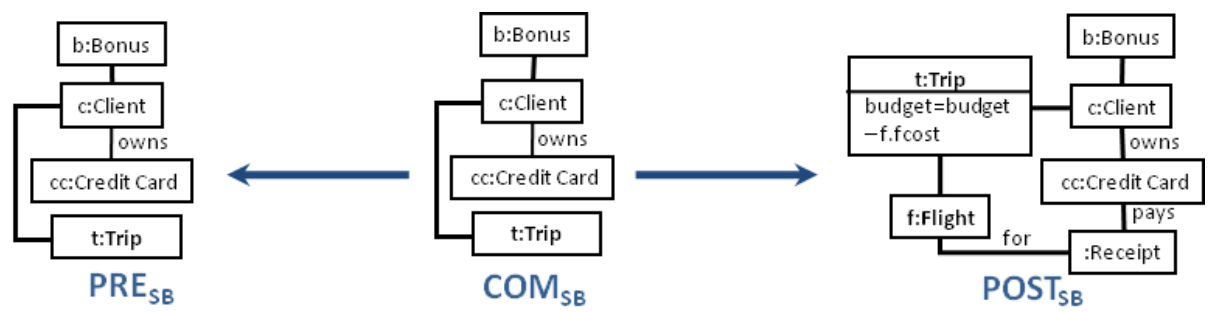

Conditions $\left(\alpha_{c}\right)$ :

t.min_dep $<$ f.dep $<$ t.max_dep,t.min_arr $<$ f.arr $<$ t.max_arr.

Fig. 6. Shared behaviour operation of goal and Prov1::FlightRes of Fig. 5 
We may consider several special kinds of partial matches which are of interest.

Definition 3. (classes of partial matches) Given a partial match $m$ as above:

- $m$ provides positive progress if post $_{C}$ is not an isomorphism (or, equivalently, if post $_{S B}$ is not an isomorphism).

- $m$ provides negative progress if pre $_{S B}$ is not an isomorphism.

- $m$ provides progress if $m$ provides positive progress or negative progress.

- $m$ is demanding if $m_{P R E_{P}}$ is not an isomorphism.

- $m$ is weakly complete if $m_{P O S T_{R}}$ is an isomorphism.

- $m$ is complete if diagram (3) is a pullback, $m_{P R E_{R}}$ and pre $e_{R}$ are jointly surjective, and $m_{P O S T_{R}}$ is an isomorphism.

Two completely unrelated rules may be bound by a trivial partial match. For instance, a partial match where the common rule is empty, or if the precondition and the postcondition of the common rule coincide. In this sense, the first three cases describe partial matches where the provider's rule satisfies partially some of the goals of the requester. In particular, if $m$ provides positive progress this means that the provider's rule produces some of the elements that the requester asks to be produced. Similarly, if $m$ provides negative progress then the provider's rule consumes some of the elements that the requester asks to be consumed. Finally, $m$ provides progress if it provides any progress at all.

If $m$ is weakly complete then this means that the provider's rule produces all the elements that are asked by the requester but it may not consume all the elements that are specified to be consumed. If $m$ is complete and not demanding this means that the provider's rule fully satisfies the requester's needs, i.e. $m$ is a match. A partial match is demanding if the provider's rule demands the requester to strengthen its precondition. Conversely, this means that if $m$ is not demanding then the provider's precondition is embedded in the requester's precondition, which means that the former can be considered stronger than the latter. This kind of situation may be part of a negotiation between the provider and the requester: the contract defined by the requester has specified some resources to satisfy his needs, but the provider is answering that, to satisfy this needs more resources are needed. If $m$ is weakly complete then the provider's rule produces everything that the requester's rule asks to be produced. So this means that the requester's postcondition is embedded in the provider's postcondition. However, notice that this not necessarily means that the provider's rule consumes everything that the requester's rule asks to be consumed. This only happens if, in addition, diagram (3) is a pullback and $m_{P R E_{R}}$ and pre $_{R}$ are jointly surjective, i.e. $m$ is complete. In particular, the condition that diagram (3) is a pullback ensures that a common element cannot be preserved by $O p_{P}$ and be deleted by $O p_{R}$, and the condition that $m_{P R E_{R}}$ and pre $_{R}$ ensures that there are no elements in $P R E_{R}$ which are deleted by $O p_{R}$ and which are not common elements (and, hence, cannot be deleted by $\left.O p_{P}\right)$. 
Remainder of Requester Operation. If the match is not complete, then we may want to know what remains to be done to satisfy the rest of the requester's needs. In particular, the provider may want to use other operations to satisfy their requirements. This can be done by computing what we call the remainder of the requester's rule with respect to the shared behaviour rule.

Definition 4. (Remainder of an operation) Given operation specifications $O p_{R}=\left\langle P R E_{R} \Rightarrow P O S T_{R}, \alpha_{R}\right\rangle$ and $O p_{P}=\left\langle P R E_{P} \Rightarrow P O S T_{P}, \alpha_{P}\right\rangle$, we define the remainder of $O p_{R}$ with respect to $O p_{P}$ and a partial match $m$ as the operation $\left\langle P R E_{\text {Rem }} \Rightarrow P O S T_{R}, \alpha_{R}\right\rangle$, where $P R E_{R e m}$ is the result of applying the operation $O p_{S B}$ to $P R E_{R}$ with match $m_{P R E_{R}}$.

The idea is quite simple. We know that $P R E_{R}$ denotes the class of states where $O p_{R}$ is expected to be applicable, but also that $O p_{S B}$ specifies the shared behaviour of $O p_{P}$ and $O p_{R}$, i.e., all the deletions and additions which are shared by both operations. Then, $P R E_{\text {Rem }}$ would describe the states after these deletions and additions, and $\left\langle P R E_{R e m} \Rightarrow P O S T_{R}, \alpha_{R}\right\rangle$ would specify the effects that are yet to be implemented by another provider operation.

For example in Fig. 5, the left-hand side $P R E_{R e m}$ of the remainder rule is obtained by applying the shared behaviour suboperation (shown in Fig. 6) to the left-hand side $P R E_{R}$ of the goal, while the remainder's postcondition is $P O S T_{R}$. $O p_{R e m}$ may be matched with Prov 2 : HotelRes in the same way, leaving empty remainder.

It is not difficult to prove that the remainder is the trivial operation, i.e. $P R E_{\text {Rem }}=P O S T_{R}$, if and only if the match $m$ is complete. Moreover, we
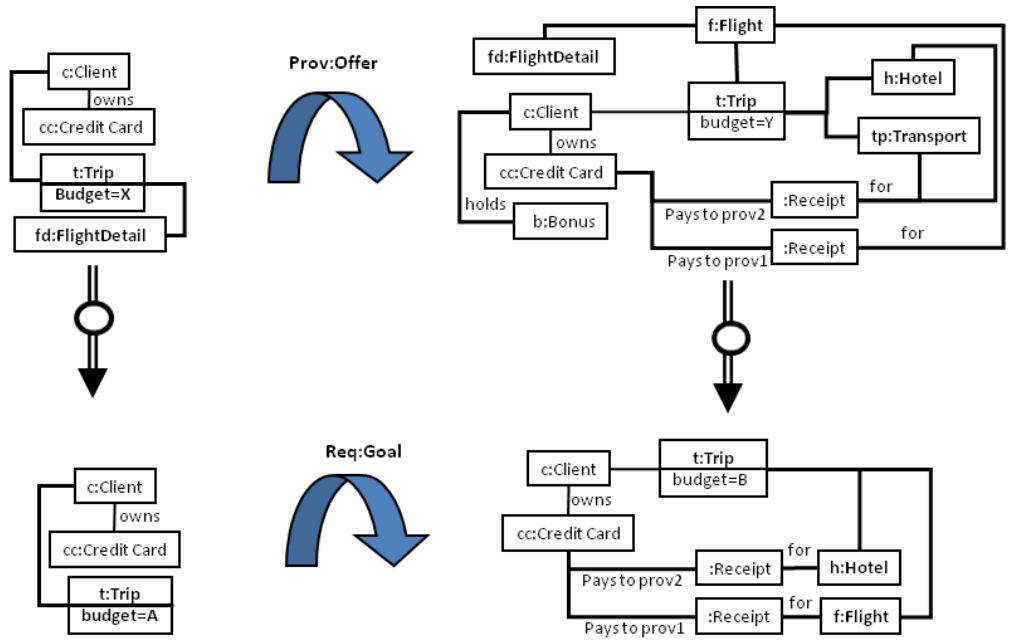

Constraints:

h.dep = f.dep, t.min_dep $\leq$ f.dep $\leq$ t.max_dep,t.min_dep $\leq$ h.dep $\leq$ t.max_dep, h.arr $=$ f.arr, t.min_arr $\leq$ f.arr $\leq$ t.max_arr, t.min_arr $\leq$ h.arr $\leq$ t.max_arr, $Y=X-$ f.fcost-h.hcost-tp.tcost, B=A-h.hcost-f.fcost.

Fig. 7. Composed Operation of both Provider Offers 
can also prove that if a provider operation $O p_{P}^{1}$ can be partially matched via $m_{1}$ to a request $O p_{R}$ leaving $O p_{R e m}$ as a remainder, and if another provider operation $O p_{P}^{2}$ can be partially matched via $m_{2}$ to $O p_{R e m}$ leaving as a new remainder $O p_{R e m}^{\prime}$, then we can compose $O p_{P}^{1}$ and $O p_{P}^{2}$ to form a new operation $O p_{P}^{3}=\left\langle P R E_{P}^{3} \Rightarrow P O S T_{P}^{3}, \alpha_{P}^{3}\right\rangle$ that can be partially matched to $O p_{R}$ via $m_{3}$, which is built from $m_{1}$ and $m_{2}$, directly leaving as a remainder $O p_{R e m}^{\prime}$. This means that the global effect of this new operation is the same one as the sequential application of $O p_{P}^{1}$ and $O p_{P}^{2}$. In particular, this means that if $m_{2}$ is complete then $m_{3}$ is also complete.

The operation $O p_{P}^{3}$ is built analogously to the so-called concurrent rule for the consecutive application of two graph transformation rules [16: Intuitively, $P R E_{P}^{3}$ is the union of $P R E_{P}^{1}$ and $\left(P R E_{P}^{2}\right.$ minus $\left.P O S T_{P}^{1}\right)$ and $P O S T_{P}^{3}$ is the union of $P O S T_{P}^{2}$ and $\left(P O S T_{P}^{1}\right.$ minus $\left.P R E_{P}^{2}\right)$, where in each case elements from different graphs are identified if they are mapping to the same elements of the requester specification. As for conditions, $\alpha_{P}^{3}$ is the conjunction of $\alpha_{P}^{1}, \alpha_{P}^{2}$, and all equations $x_{1}=x_{2}$, for all variables $x_{1}$ from $\alpha_{P}^{1}$ and $x_{2}$ from $\alpha_{P}^{2}$ which are bound to the same variable $x$ from $\alpha_{R}$ via $m_{1}$ and $m_{2}$, respectively. For our example, Fig. 7 shows the resulting composed operation.

Finding Complete Solutions. We describe a resolution-like procedure for building a complete match to a requester specification. This procedure is terminating, correct and complete, i.e., given a requester operation $O p_{R}$, if at all possible this procedure will combine suitable provider operations into a single composed one which forms a complete match for the requestor's goal. Moreover, this is done in a finite number of steps. The procedure is presented as an inference rule whose application is non-deterministic, although in practice we could use heuristics to guide the search and to produce first the results which are considered better according to some criteria.

Let us describe this procedure in detail. We describe the computation states of our procedure as 3 -tuples $\left\langle O p_{P}, m, O p_{R e m}\right\rangle$, where $O p_{P}$ is a provider operation (perhaps built from more basic operations), $m$ is a partial match from $O p_{P}$ to $O p_{R}$, and $O p_{R e m}$ is the remainder associated to $m$. Intuitively, $O p_{P}$ represents the partial solution that we have built up to that point, $m$ is the partial match that tells us in which way $O p_{P}$ partially satisfies the request, and $O p_{R e m}$ is the the part of the request that we still have to satisfy. In this context, we consider that the initial state is the 3-tuple $\left\langle\right.$ Triv, triv, $\left.O p_{R}\right\rangle$, where Triv is the trivial (empty) operation, triv is the trivial empty match and, obviously, the remaining part to satisfy is the whole request. Then, the procedure is based on the following inference rule:

$$
\frac{\left\langle O p_{P}^{1}, m_{1}, O p_{R e m}^{1}\right\rangle}{\left\langle O p_{P}^{2}, m_{2}, O p_{R e m}^{2}\right\rangle}
$$

If there is a provider operation $O p_{P}^{3}$, and a partial match $m_{3}$ from $O p_{P}^{3}$ to $O p_{\text {Rem }}^{1}$, such that $m_{3}$ provides progress, $O p_{R e m}^{2}$ is the remainder associated to $m_{3}$, and $O p_{P}^{2}$ and $m_{2}$ are, respectively, the composition of $O p_{R e m}^{1}$ and $O p_{\text {Rem }}^{3}$ and the associated partial match from $O p_{P}^{2}$ to $O p_{R}$. 
An execution of this procedure is a sequence:

$$
\left\langle\text { Triv, triv }, O p_{R}\right\rangle \Longrightarrow\left\langle O p_{P}^{1}, m_{1}, O p_{\text {Rem }}^{1}\right\rangle \Longrightarrow \ldots \Longrightarrow\left\langle O p_{P}^{n}, m_{n}, O p_{\text {Rem }}^{n}\right\rangle
$$

where, for each $i,\left\langle O p_{P}^{i+1}, m_{i+1}, O p_{R e m}^{i+1}\right\rangle$ can be inferred from $\left\langle O p_{P}^{i}, m_{i}, O p_{R e m}^{i}\right\rangle$. Then, an execution is successful if the final match $m_{n}$ is complete.

It is not difficult to show that the above procedure is correct, complete and terminating. In particular, it is sound in the sense, for every $i, m_{i}$ is a partial match from $O p_{P}^{i}$ to $O p_{R}$, and $O p_{\text {Rem }}^{i}$ is the corresponding remainder. It is complete in the sense that if there is a way of satisfying completely the request by applying a sequence of provider operations then there exists an execution that will return a composed operation, together with a complete match. Finally the procedure is terminating, i.e. there are no executions of infinite length and, moreover, there is a finite number of executions, provided that the graphs involved are finite and that there is a finite number of provider operations. This is due to the fact that the number of additions and deletions requested in a goal is finite. Since we are assuming that all the matchings involved provide progress, the length of each execution is bounded by the number of additions and deletions specified in the request. Moreover, with a finite number of provider operations and finite graphs only, there is a finite number of partial matches between requester operation and provider operations.

\section{Conclusion}

In this paper, we have proposed an approach to the incremental composition of services using visual specifications of pre- and postconditions. The procedure is based on the repeated partial matching of provider offers with a requestor goal, which is reduced in the process until all requirements are satisfied or there are no more offers to consider. As a result, the procedure constructs a combined offer, which can be presented to the requestor to confirm if it is acceptable.

The formalization of these notions and constructions is provided in the appendix for information. In summary, the main theoretical results are as follows.

1. A definition of partial matching allowing the comparison of individual offers of services with the global goal of the requestor.

2. An incremental matching procedure based on the construction of a remainder of a goal with respect to a chosen partial match. Assuming a finite number of offers, the incremental matching procedure terminates. Thus, partial matching is decidable.

3. Each combined offer constructed as result of the matching has the same overall effect as executing the sequence of offers from which the combined offer is derived. That means, for each sequence of applications of individual offer rules there exists an application of the combined offer rule with the same effect, and vice versa. 
In general, there will be several combined offers computed for a given request. These could be presented to the client to let them choose the most suitable one. Alternatively, the selection could be automated based on a specification of preferences (non-functional properties) by the client. Once an offer is computed it can be stored in the repository of services, such that new requests can be served more quickly, matching them against existing combined offers. Future work will address the use of non-functional requirements for the selection of offers, as well as a proof-of-concept implementation of the approach.

\section{References}

1. Papazoglou, M.P.: Service-oriented computing: Concepts, characteristics and directions. In: Fourth International Conference on Web Information Systems Engineering (WISE 2003), Roma, Italy, December 2003, IEEE Computer Society, Los Alamitos (2003)

2. Kim, I.W., Lee, K.H.: Describing semantic web services: From UML to OWL-S. In: IEEE International Conference on Web Services ICWS 2007, Salt Lake City, UT, USA, July 2007, pp. 529-536. IEEE Computer Society, Los Alamitos (2007)

3. de Bruijn, J., Lausen, H., Polleres, A., Fensel, D.: The web service modeling language WSML: An Overview. In: Sure, Y., Domingue, J. (eds.) ESWC 2006. LNCS, vol. 4011, pp. 590-604. Springer, Heidelberg (2006)

4. Hausmann, J.H., Heckel, R., Lohmann, M.: Model-based development of web service descriptions: Enabling a precise matching concept. International Journal of Web Services Research 2(2), 67-84 (2005)

5. Peltz, C.: Web services orchestration and choreography. Computer, 46-52 (2003)

6. van der Aalst, W.: Don't go with the flow: Web services composition standards exposed. IEEE Intelligent Systems 18(1), 72-76 (2003)

7. Sirin, E., Hendler, J., Parsia, B.: Semi-automatic composition of web services using semantic descriptions. In: Workshop on Web Services: Modeling, Architecture and Infrastructure, pp. 17-24 (2002)

8. Wu, D., Parsia, B., Sirin, E., Hendler, J.A., Nau, D.S.: Automating DAML-S web services composition using SHOP2. In: Fensel, D., Sycara, K., Mylopoulos, J. (eds.) ISWC 2003. LNCS, vol. 2870, pp. 195-210. Springer, Heidelberg (2003)

9. Stollberg, M., Keller, U., Lausen, H., Heymans, S.: Two-phase web service discovery based on rich functional descriptions. In: Franconi, E., Kifer, M., May, W. (eds.) ESWC 2007. LNCS, vol. 4519, pp. 99-113. Springer, Heidelberg (2007)

10. Klusch, M., Kaufer, F.: Wsmo-mx: A hybrid semantic web service matchmaker. Web Intelli. and Agent Sys. 7(1), 23-42 (2009)

11. Heckel, R., Cherchago, A.: Structural and behavioral compatibility of graphical service specifications. Journal of Logic and Algebraic Programming 70(1.1), 15-33 (2007)

12. Kreger, H.: Web services conceptual architecture, IBM Software Group (2001), http://www.ibm.com

13. Orejas, F.: Attributed graph constraints. In: Ehrig, H., Heckel, R., Rozenberg, G., Taentzer, G. (eds.) ICGT 2008. LNCS, vol. 5214, pp. 274-288. Springer, Heidelberg (2008) 
14. Ehrig, H., Ehrig, K., Prange, U., Taentzer, G.: Fundamentals of Algebraic Graph Transformation. Monographs in Theoretical Computer Science. An EATCS Series. Springer, Heidelberg (2006)

15. Heckel, R., Llabrés, M., Ehrig, H., Orejas, F.: Concurrency and loose semantics of open graph transformation systems. Mathematical Structures in Computer Science 12, 349-376 (2002)

16. Rozenberg, G. (ed.): Handbook of graph grammars and computing by graph transformation. Concurrency, parallelism, and distribution, vol. 3. World Scientific Publishing Co., Inc., River Edge (1999) 